\title{
Tratamento da Deficiência do Hormônio de Crescimento (GH) em Crianças: Comparação entre o Uso de Canetas Versus Frascos/Seringas para a Aplicação do GH
}

\begin{abstract}
RESUMO
O objetivo deste estudo foi comparar as duas apresentações de GH recombinante humano ( $\mathrm{rGH}$ ) para tratamento da deficiência de $\mathrm{GH}(\mathrm{DGH})$. Dez crianças pré-púberes portadoras de DGH foram acompanhadas durante 6 meses. Elas receberam, por 3 meses, injeções com seringa e, a seguir, com canetas por mais 3 meses. A aceitabilidade foi avaliada através de questionário. O desperdício foi calculado através da diferença entre o número de frascos/refis utilizados e o previsto para o período. A resposta ao tratamento foi avaliada pelo ganho em desvio-padrão (DP) de altura medido a cada 3 meses. Após 6 meses, 90\% dos pacientes/familiares afirmaram preferir a caneta em termos de facilidade técnica e dor local, e todos consideraram a caneta melhor em termos de facilidade de transporte e armazenamento. O desperdício foi menor com a caneta, assim como o custo. Concluímos que a administração de rGH através de caneta é mais conveniente, melhor aceita pelos pacientes e resulta em menor desperdício quando comparada com o tratamento por seringa. (Arq Bras Endocrinol Metab 2007;51/7:1093-1096)
\end{abstract}

Descritores: Tratamento com GH; Deficiência; Hormônio de crescimento; Caneta

\section{ABSTRACT}

Growth Hormone (GH) Deficiency Treatment in Children: Comparison Between Uses of Pen Versus Bottles/Syringes on GH Administration.

The aim of this study was to compare two preparations of recombinant human $\mathrm{GH}(\mathrm{rGH})$ in the treatment of $\mathrm{GH}$ deficient patients. Ten prepubertal $\mathrm{GH}$-deficient children were followed during 6 months. They received injections with syringe for 3 months, followed by pen administration for the subsequent 3 months. Acceptability was evaluated through a questionnaire. Waste of medication was calculated by the difference between the number of used bottles or refills and the calculated amount for the period. Treatment response was evaluated by SDS gain of height measured each 3 months. After 6 months, 90\% of patients/family members declared they preferred the pen regarding technical facility and local pain, and all patients considered the pen easier to transport and store. The waste of medication was lower with pen administration, as was the final cost. We concluded that pen-administered rGH treatment is more convenient, better accepted by the patients, and leads to less waste of medication when compared to the syringe administration. (Arq Bras Endocrinol Metab 2007;51/7:1093-1096)

Keywords: Treatment; Children; Deficiency; Growth hormone; Pen artigo original

\author{
FREDERICO G. MARCHISOTII \\ LUCIANI R.S. CARVALHO \\ KARINA BERGER \\ IVO J.P. ARNHOLD \\ BERENICE B. MENDONÇA
}

Unidade de Endocrinologia do Desenvolvimento, Laboratório de Hormônios e Genética Molecular - LIM/42, Hospital das Clínicas da FMUSP, São Paulo, SP.

Recebido em 24/03/06 Revisado em 06/03/07 Aceito em 13/04/07 
A

BAIXA ESTATURA É UMA CONDIÇÃO prevalente na população e entre as suas causas está a deficiência de hormônio de crescimento (DGH). A DGH é tratada através da reposição do hormônio de crescimento recombinante humano ( $\mathrm{rGH})$.

Atualmente, há no mercado brasileiro duas formas de se administrar o hormônio de crescimento: 1) frascos para diluição e posterior aplicação através de seringas e 2) canetas com a medicação em solução pronta para aplicação. No Sistema Único de Saúde (SUS), o produto atualmente adquirido deve ser aplicado através de seringas. A não utilização de canetas, pelo SUS, é atribuída ao maior custo da medicação.

Nosso objetivo foi comparar as duas formas de aplicação do rGH em um mesmo grupo de pacientes com hipopituitarismo, analisando a aceitabilidade dos pacientes e familiares, desperdício, resposta ao tratamento e o custo do tratamento.

\section{MATERIAIS E MÉTODOS}

Foram selecionados 12 pacientes pré-púberes portadores de déficit de hormônio de crescimento isolado ou associado a outras deficiências hormonais hipofisárias. O estudo foi aprovado pela comissão de ética do Hospital das Clínicas da Faculdade de Medicina da Universidade de São Paulo.

Os pacientes foram acompanhados durante 3 meses em uso de seringas e 3 meses em uso de canetas para aplicação de rGH. Foram obtidos os dados auxológicos e dosados os seguintes hormônios: IGF1, IGFBP3, LH, FSH, Testosterona/Estradiol, TSH, T4, T3, T4 livre nos tempos 0,3 e 6 meses de acompanhamento.

A medicação foi fornecida pela NovoNordisk (Bagvsaerd / Denmark) nas seguintes apresentações: frascos de $4 \mathrm{U}$ (Norditropin) e canetas com refis de $10 \mathrm{mg}$ (Norditropin Simplexx). A técnica de aplicação foi orientada por funcionária especializada da empresa, juntamente com a equipe de enfermagem do hospital. Todos os pacientes usaram regularmente a medicação, sendo tolerado apenas um total de 15 dias sem uso, nos 6 meses de acompanhamento, como critério de exclusão.

Foi desenvolvido e fornecido um questionário aos pacientes/familiares no início do protocolo e, após 3 meses, data da introdução do uso da caneta, foram fornecidos dois outros questionários. Os pais foram orientados quanto às perguntas e as dúvidas foram respondidas, se porventura existissem. Os questionários foram aplicados para avaliar os seguintes itens relativos à medicação: técnica de aplicação, dor local, armazenamento e transporte, aderência ao tratamento e preferência. Também foi solicitado aos pacientes que anotassem o número de frascos e refis utilizados durante o período de acompanhamento, para que calculássemos o desperdício. A resposta ao tratamento foi avaliada através do ganho em DP de altura medido a cada 3 meses através de estadiômetro com precisão milimétrica (média de 3 medidas). O custo do tratamento foi calculado através dos valores das medicações, usadas no estudo, fornecidos pelo SAR (serviço de distribuição de medicamentos especiais / de alto custo), e a diferença de valor expressa em porcentagem (\%). Os preços utilizados para os cálculos foram preços não promocionais.

\section{RESULTADOS}

Dois pacientes foram retirados do estudo, 30 e 40 dias após o início do mesmo, devido à irregularidade no tratamento. Ambos estavam em uso de seringas na época. Foram analisados os dados de dez pacientes prépúberes, sob tratamento com rGH aplicado na forma de seringas, numa dose de $0,03 \mathrm{mg} / \mathrm{kg} / \mathrm{dia} \quad(0,1$ $\mathrm{U} / \mathrm{kg} /$ dia) ao iniciar o estudo. Sete eram do sexo feminino. Quatro apresentavam déficit de $\mathrm{GH}$ isolado e seis, déficit hipotálamo/hipofisário múltiplo. As outras deficiências hormonais hipotálamo/hipofisárias, quando presentes, estavam adequadamente repostas. No início do estudo, a idade cronológica (média \pm DP) $=7,6 \pm 3,7$ anos, idade óssea $=5,41 \pm 3,7$ anos, altura $=-1,52 \pm 1,6 \mathrm{DP}$ e peso $=-0,5 \pm 1,2 \mathrm{DP}$. As crianças estavam em tratamento, em média, há 2,68 anos.

Uma paciente apresentou desidratação por falha do uso de DDAVP (acetato de desmopressina) durante o acompanhamento. Não houve outras intercorrências.

Os resultados relativos à aceitabilidade da medicação estão resumidos na tabela 1 .

Tabela 1. Avaliação do manuseio das duas apresentações pelos pacientes.

\begin{tabular}{lcc}
\hline Técnica de aplicação & Seringa & Caneta \\
Muito fácil & 1 & 7 \\
Fácil & 5 & 3 \\
Difícil & 4 & 0 \\
Muito difícil & 0 & 0 \\
Dor à aplicação & & \\
Nenhuma & 2 & 5 \\
Pouca dor & 5 & 5 \\
Dor moderada & 3 & 0 \\
Muita dor & 0 & 0 \\
Desperdício da medicação & & \\
Não ocorre & 1 & 5 \\
Pequena & 6 & 5 \\
Média & 3 & 0 \\
Grande & 0 & 0 \\
Armazenamento e transporte & & \\
Muito fácil & 1 & 6 \\
Fácil & 4 & 3 \\
Difícil & 5 & 1 \\
Muito difícil & 0 & 0 \\
\hline
\end{tabular}

Os números na tabela representam o número de pacientes que escolheram a opção. 
Todos os pacientes consideraram a técnica de aplicação da caneta muito fácil ou fácil, enquanto $40 \%$ dos pacientes consideraram a técnica de aplicação através de seringas difícil.

Todos os pacientes atestaram nenhuma ou pouca dor após aplicação via caneta, enquanto que $30 \%$ dos pacientes reclamaram de dor moderada após aplicação através de seringas.

Todos os pacientes informaram nenhuma ou pequena perda da medicação durante uso de canetas; porém, 30\% dos pacientes informaram perda moderada da medicação durante o uso de frascos e seringas.

Apenas um paciente declarou ser difícil o armazenamento e transporte da caneta, enquanto $50 \%$ dos pacientes consideraram difícil o armazenamento e transporte de frascos e seringas. $O$ paciente que analisou este item negativamente após o uso da caneta também o fez após o uso de frascos e seringas.

Todos os pacientes relataram ter esquecido nenhuma ou poucas vezes de aplicar a medicação nos 6 meses de acompanhamento. Nenhum paciente relatou ter esquecido ou não aplicado a medicação por outro motivo qualquer por um período maior que 15 dias.

Quando questionados diretamente quanto à preferência, $100 \%$ dos pacientes preferiram o uso de canetas (tabela 2). Todos consideraram a caneta mais fácil de aplicar, transportar e armazenar, e causadora de menor desperdício. Um paciente relatou o mesmo nível de dor com o uso da caneta ou da seringa, os outros $90 \%$ consideraram a caneta menos dolorosa.

Houve um ganho médio de 0,21 DP de altura nos 3 primeiros meses (uso de frascos/seringas) e de 0,21 DP nos 3 meses seguintes (uso de caneta), ou seja, não houve diferença no ganho de altura ao compararmos a forma de aplicação da medicação. O tempo de observação foi insuficiente para que se tenha uma conclusão definitiva sobre a resposta terapêutica.

Tabela 2. Respostas dos pacientes à comparação entre as duas formas de aplicação do GH.

\begin{tabular}{lcc}
\hline Mais fácil & caneta & 10 \\
Menos dor* & seringa & 0 \\
& caneta & 9 \\
Menor desperdício & seringa & 0 \\
\multirow{2}{*}{ Mais fácil transportar / armazenar } & caneta & 10 \\
& seringa & - \\
Preferência & caneta & 10 \\
& seringa & - \\
& caneta & 10 \\
\hline
\end{tabular}

Os números nas tabelas representam o número de pacientes que escolheram a opção.

* Um paciente relatou sentir o mesmo grau de dor com os dois métodos de aplicação.
Para o tratamento de 10 pacientes, foi utilizado um total de 599 frascos, sendo que 40 (6,7\%) destes por desperdício; assim, houve um desperdício médio de 4 frascos $(5,33 \mathrm{mg}) / 3$ meses por paciente.

Foi utilizado um total de 86 refis, sendo 1 $(1,16 \%)$ destes por desperdício; assim, houve um desperdício médio de 0,1 refil $(1 \mathrm{mg}) / 3$ meses por paciente.

O uso de canetas propiciou uma economia total (já somada o custo derivado do desperdício) de cerca de $12 \%$ em relação ao uso de frascos/seringas, após 3 meses de tratamento. Não foi considerado o valor da seringa no cálculo.

Os níveis séricos dos hormônios tireoidianos se mantiveram normais durante todo o estudo, e os níveis dos esteróides sexuais se mantiveram em valores prépuberais (tabela 3 ).

\section{DISCUSSÃo}

Desde 1989, para tornar mais fácil para os pacientes e pais lidar com o grande número de injeções, derivado da aplicação diária do $\mathrm{rGH}$, um sistema de injeção através de canetas com agulhas menores, inicialmente usado para aplicação de insulina, foi estudado em portadores de déficit de GH (1). Gluckman e Cutfield (2) observaram que os pacientes preferiam o sistema de caneta, devido ao menor tempo para administração da medicação, preparo menos complexo, maior liberdade para viajar e maior independência. No mesmo estudo, foi observado que crianças maiores de 10 anos eram mais aptas a se auto-aplicar a medicação na forma de caneta.

A Nova Zelândia foi o primeiro país a licenciar esse sistema de aplicação. Atualmente, é o sistema mais usado nos países desenvolvidos. Ainda hoje, no Brasil, a forma de aplicação do rGH pela rede pública é feita através de frascos/seringas.

Tabela 3. Valores hormonais séricos durante o estudo.

\begin{tabular}{lccc}
\hline & Basal & $\mathbf{3}$ meses & $\mathbf{6}$ meses \\
IGF-1 (DP) & $0,1 \pm 1,3$ & $1,0 \pm 1,0$ & $0,7 \pm 1,8$ \\
IGFBP3 (DP) & $0,3 \pm 1,7$ & $0,8 \pm 1,0$ & $0,8 \pm 2,1$ \\
TSH $(\mu \mathrm{U} / \mathrm{ml})$ & $1,7 \pm 1,6$ & $1,3 \pm 1,4$ & $1,2 \pm 1,1$ \\
T4 $(\mu \mathrm{g} / \mathrm{dl})$ & $8,4 \pm 2,6$ & $8,8 \pm 2,8$ & $8,1 \pm 2,8$ \\
T3 $(\mathrm{ng} / \mathrm{dl})$ & $166 \pm 22,6$ & $173 \pm 11,2$ & $160 \pm 34,5$ \\
T4 livre (ng/dl) & $1,1 \pm 0,47$ & $1,1 \pm 0,5$ & $0,9 \pm 0,2$ \\
LH (UI/L) & $1,0 \pm 1,8$ & $0,3 \pm 0,2$ & $1,0 \pm 2,0$ \\
FSH (Ul/L) & $2,1 \pm 3,8$ & $0,8 \pm 0,6$ & $1,5 \pm 2,3$ \\
Estradiol $(\mathrm{pg} / \mathrm{mL}) *$ & $<13$ & $<13$ & $<13$ \\
Testosterona $(\mathrm{ng} / \mathrm{dL}) *$ & $<14$ & $<14$ & $<14$
\end{tabular}

Valores apresentados na forma de média \pm DP.

* Valores verificados em todos pacientes. 
No nosso estudo, a grande maioria (> 90\%) dos pacientes avaliou positivamente o sistema de aplicação através de caneta em relação a frascos/seringas em todos os itens de comparação. A administração de hormônio de crescimento através de canetas é mais conveniente, melhor aceita pelos pacientes e o desperdício da medicação, aplicada via seringa, foi cerca de seis vezes maior quando comparado à aplicação via caneta.

A aderência dos pacientes é um fator-chave para o resultado de um tratamento de longo prazo e que requer uso de via parenteral. Em um estudo com 103 crianças, Stanhope e cols. (3) observaram que a reconstituição da medicação foi o principal fator da falta de aderência. A reconstituição pode ser evitada usando-se a formulação líquida presente nas canetas. A formulação líquida, cancelando a necessidade de diluição prévia, tornou a aplicação do $\mathrm{rGH}$ mais conveniente e menos sujeita a erro de dosagem. Além disso, ao diminuir a manipulação da droga, ela provavelmente reduz a chance de contaminação da solução ou do equipamento de injeção, complicação que já foi observada por Zweens e cols. (4). Outra vantagem, percebida pelos pacientes, é a pequena freqüência de troca dos refis, devido à maior concentração da medicação. Essa maior concentração do $\mathrm{rGH}$, correspondendo a um menor volume de injeção, poderia levar a uma redução da biodisponibilidade, como mostrado para insulina em alguns estudos (5-7). No entanto, a biodisponibilidade da droga, através do uso de canetas, foi comparada no passado com a mesma aplicada através de frascos/seringas, mostrando resultados semelhantes $(1,8)$.

No passado, o preço da caneta de aplicação do rGH era um fator limitante ao tratamento com este dispositivo. Atualmente, com o sistema de canetas descartáveis, mais baratas, poderia ser indicado o uso do rGH na forma de caneta ao invés de frascos/seringas. Além disso, observa-se uma tendência ao abandono da fabricação e comercialização, pela indústria farmacêutica, do rGH na forma de frascos.

Concluímos que a administração de rGH através de caneta é mais conveniente, melhor aceita pelos pacientes, e resulta em menor desperdício quando comparado ao tratamento por seringa, devendo o seu uso ser instituído pelo sistema público de saúde no Brasil.

\section{AGRADECIMENTOS}

Ao Laboratório de Hormônios e Genética (LIM 42) do Hospital das Clínicas - FMUSP, pela realização das dosagens hormonais.

À NovoNordisk do Brasil, por fornecer a medicação utilizada no estudo.

A NovoNordisk Ltda. apenas contribuiu com o fornecimento da medicação (rhGH) nas duas formas de apresentação usadas (frascos e canetas) no estudo. Não houve participação ou qualquer interferência na elaboração do projeto, coleta e interpretação de dados ou redação do manuscrito.

\section{REFERÊNCIAS}

1. Jorgensen JO, Mooller J, Jensen FS, Joorgensen JT, Christiansen JS. Growth hormone administration by means of an injection pen. Pharmacol Toxicol 1989;65:96-9.

2. Gluckman PD, Cutfield WS. Evaluation of a pen injector system for growth hormone treatment. Arch Dis Child 1991;66:686-8.

3. Stanhope R, Buchanan C, Butler G, Costigan C, Dunger D, Greene $S$, et al. An open-label acceptability study of Norditropin SimpleXX - a new liquid growth hormone formulation. J Pediatr Endocrinol Metab 2001;14:735-40.

4. Zweens MJ, Drayer NM, Dankert J. Bacterial contamination of growth hormone solution and injection equipment during use by growth hormone deficient children. $J$ Hosp Infect 1991; 18:123-30.

5. Binder C. Absorption of injected insulin. A clinical-pharmacological study. Acta Pharmacol Toxicol (Copenh) 1969;2:1-84.

6. Hildebrandt $P$, Sestoft $L$, Nielsen $S L$, Nielsen SL. The absorption of subcutaneously injected short-acting soluble insulin: influence of injection technique and concentration. Diabetes Care 1983;6:459-62.

7. de Meijer PHEM, Russel FGM, Van Lier HJJ, Van Ginneken CAM. A comparison of three mathematical models to describe the disappearance curves of subcutaneously injected ${ }^{125}$ |-labelled insulin. Brit J Clin Pharmacol 1989;27:461-

8. Blok GJ, van der Veen EA, Susgaard S, Larsen F. Influence of concentration and injection volume on the bioavailability of subcutaneous growth hormone: comparison of administration by ordinary syringe and by injection pen. Pharmacol Toxicol 1991;68:355-9.

\section{Endereço para correspondência:}

Frederico G. Marchisotti

Av. Dr. Enéas de Carvalho Aguiar 155, 2ํandar,

Bloco 6 (PAMB)

05403-000 São Paulo, SP

Fax: (1 1) 3069-7519

E-mail: fmarchisotti@terra.com.br 